\title{
PROGRAMAÇÃO DE ESCALA DE MAQUINISTAS PARA UMA FERROVIA DE CARGA GERAL: UMA REVISÃO DA LITERATURA
}

\author{
Maria Fernanda Vieira Zanetti \\ Instituto Militar de Engenharia \\ Mestrado em Engenharia de Transportes \\ mafezanetti@yahoo.com.br \\ Orivalde Soares da Silva Júnior \\ Instituto Militar de Engenharia \\ Mestrado em Engenharia de Transportes \\ orivalde@ime.eb.br
}

\begin{abstract}
RESUMO
As ferrovias de carga por possuírem grandes extensões, num geral, demandam tempo de trabalho dos maquinistas, por turno, superior ao tempo de viagem origem/destino do trem, o que torna necessária a troca dos maquinistas ao longo deste percurso. As trocas são feitas em pontos de destacamentos pré-determinados, onde os maquinistas se apresentam no dia e hora informados pela equipe da estação. Além disto, podem ficar à disposição da empresa em suas residências/alojamentos (sobreaviso) ou de prontidão (nas estações). A proposta deste estudo é através da pesquisa do estado da arte sobre planejamento de escala de condutores, encontrar um modelo matemático, dentre muitos já apresentados ao redor do mundo, sob diferentes circunstâncias, que possa ser aplicado total ou parcialmente, às ferrovias de carga brasileiras, de maneira a obter-se uma escala de trabalho ótima, tal que a alocação da mão-de-obra e consequente custos com horas-extras, prontidão e sobreaviso sejam minimizadas, tudo isto se levando em conta as restrições impostas pelas características da atividade, necessidades dos maquinistas, normas das empresas e legislações vigentes.
\end{abstract}

Palavras-chave: revisão da literatura; otimização; escala de maquinistas.

\begin{abstract}
The freight railways, for having large extensions, in general, require the working time of the engine driver, per shift, exceeding the journey time origin/destination of the train, which makes it necessary to exchange the engine drivers along this route. Exchanges are made at pre-determined points of secondments, where the engine drivers present themselves on the day and time informed by the station staff. In addition, they can be at the disposal of the company in their residences/lodgings (on standby) or readiness (in the stations). The purpose of this study is to research the state of the art on drivers scale, to find a mathematical model, among many already presented around the world, under different circumstances, which can be wholly or partially applied to the railways of Brazilian cargo, so as to obtain an optimum scale of work, such that the allocation of manpower and consequent costs with overtime, readiness and on standby employees are minimized, taking into account the constraints imposed by the characteristics of the activity, the needs of the drivers, the norms of the companies and legislation in force.
\end{abstract}

Keywords: literature review; optimization; engine drivers timetable.

\section{INTRODUÇ̃̃O}

$\mathrm{Na}$ literatura, diversos trabalhos têm surgido no que diz respeito a problemas de roteirização e escala (routing and scheduling problems). Estes permitem aos diversos modais o gerenciamento em nível de operação dos veículos (trens de carga, passageiros, aviões, automóveis, caminhões) e seus respectivos condutores.

Como planejamento de escalas, espera-se que o modelo matemático construa e atribua viagens aos veículos/condutores/tripulação, as quais deverão ser executadas por estes, de modo a respeitar as restrições impostas, sejam estas de fins legais, sindicais ou normativos. Já na alocação, espera-se que uma sequência de trabalhos seja construída para cada dia da semana, atribuindo cada uma destas sequências a um condutor, de maneira a maximizar a eficiência da alocação dos condutores aos veículos e cobrir todas as viagens a serem realizadas. 
Para a maioria dos casos, tem se utilizado como base para a resolução de problemas do tipo "planejamento de escala de serviços para condutores de veículos", duas aplicações: a primeira o crew scheduling problem e a segunda o crew rostering problem. O crew scheduling problem trata do problema da alocação dos recursos, enquanto que o crew rostering problem trata do problema da escala de serviços. Elas podem ainda ser abordadas sobre duas óticas, independente do horizonte de planejamento: cíclico ou individualizado. Segundo Constantino (1997), o planejamento de escala cíclica constrói uma sequência contendo todas as jornadas diárias de trabalho intercaladas por dias de folga. Esta sequência constitui-se num ciclo de trabalho que é repetida por todos os condutores, sendo que cada um a inicia em uma posição diferente. Já o planejamento individualizado consiste em construir sequências individuais de jornadas de trabalho, levando-se em consideração o histórico de cada condutor. Normalmente, o problema de alocação está relacionado com o planejamento diário, enquanto que o problema de escala está relacionado a horizontes maiores de planejamento, ou seja, semanas ou meses.

No caso do transporte ferroviário de cargas é muito comum que o pessoal repouse fora de sua sede e outro condutor assuma o trem até o seu destino. Neste caso, as sequências de trabalho serão compostas por diversas viagens cumpridas em diversas jornadas, e separadas por períodos de folga e/ou repouso, retornando o condutor no final à sua sede base.

No Brasil, de acordo com a Associação Nacional dos Transportadores Ferroviários (ANTF, 2019), o transporte ferroviário corresponde a apenas $15 \%$ do volume de carga transportado dentro da matriz de transportes. $65 \%$ deste transporte é feito por rodovias, o que não condiz com um país de dimensões continentais como o Brasil. Isto se torna ainda mais evidente quando comparamos a matriz de transporte de cargas e a densidade da malha ferroviária brasileira disponível com as de outros países de mesmo porte territorial, conforme o observado nas Figuras 1 e 2, respectivamente, a seguir.

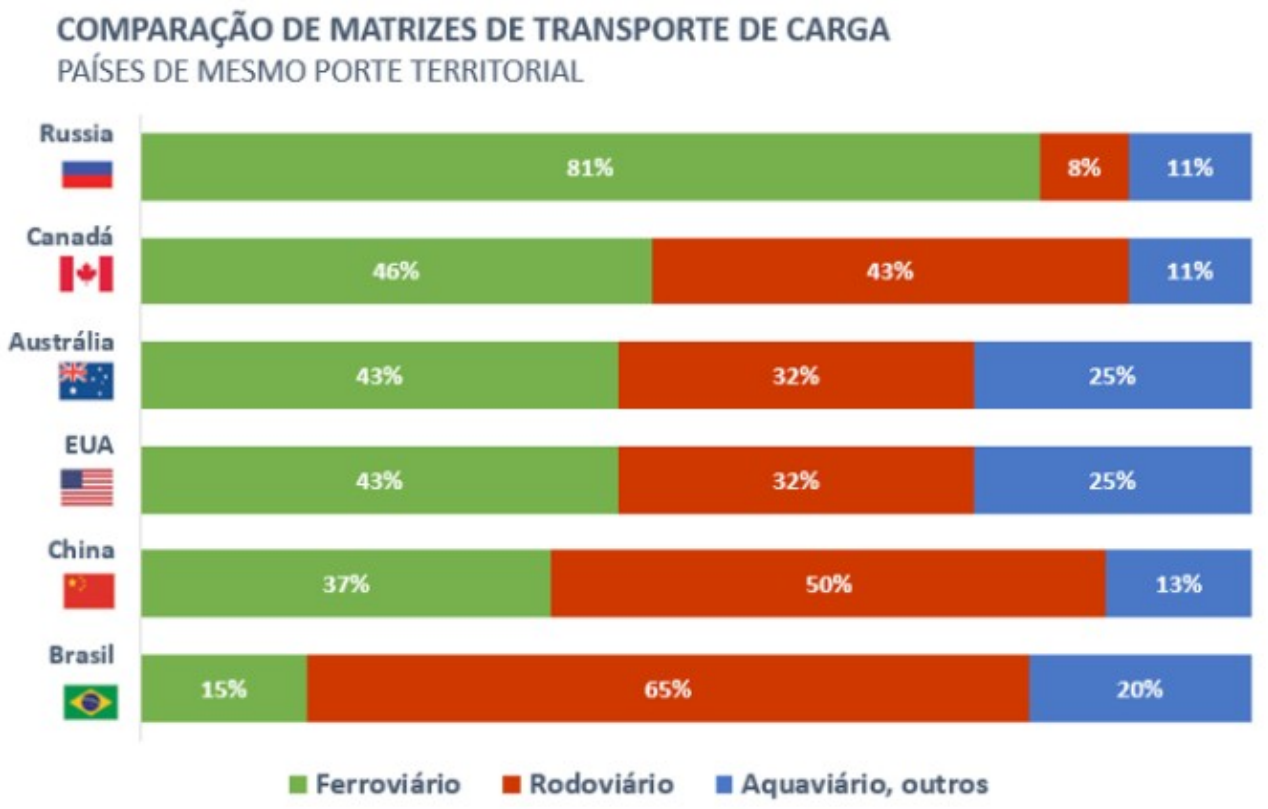

Figura 1: Comparação de matrizes de transporte de cargas em países de mesmo porte territorial que o Brasil

Fonte: ANTF (2019) 


\section{DENSIDADE DAS MALHAS FERROVIÁRIAS}

\begin{tabular}{|c|c|c|c|}
\hline & $\begin{array}{c}\text { Área } \\
\text { (milhões km²) }\end{array}$ & $\begin{array}{l}\text { Ferrovias } \\
(\text { mil km) }\end{array}$ & $\begin{array}{l}\text { Ferrovias/Áreas } \\
\left(\mathrm{km} / 1.000 \mathrm{~km}^{2}\right)\end{array}$ \\
\hline EUA & 9,83 & 293,56 & 29,8 \\
\hline Índia & 3,29 & 68,53 & 20,8 \\
\hline África do Sul & 1,22 & 20,99 & 17,2 \\
\hline Argentina & 2,78 & 36,92 & 13,3 \\
\hline China & 9,60 & 124,00 & 13,2 \\
\hline México & 1,96 & 15,39 & 7,8 \\
\hline Canadá & 9,98 & 77,93 & 7,8 \\
\hline Rússia & 17,1 & 87,16 & 5,1 \\
\hline Austrália & 7,74 & 36,97 & 4,8 \\
\hline Brasil & 8,52 & 29,18 & 3,4 \\
\hline
\end{tabular}

Figura 2: Densidade das malhas ferroviárias de países de dimensões continentais Fonte: ANTF (2019)

Mas isto está mudando. As ferrovias de carga brasileiras ampliaram significativamente o volume nelas transportado desde a época do início das concessões, em 1997. Em 10 anos teve-se um aumento de $112,5 \%$ no volume movimentado, o que significa dizer que se atingiu o recorde de 538 milhões de toneladas úteis transportadas em 2017 (ANTF, 2019).

Mas para que estes produtos cheguem aos seus destinos, são necessárias composições (trens), e estas, necessitam de maquinistas qualificados para operá-las. Num geral, a condução do trem desde a origem até o destino, necessita ser dividida por vários maquinistas. Isto se dá devido à grande extensão da malha, conforme o observado na Tabela 1, o que acarreta em ultrapassagem do período em condução do maquinista permitido pela legislação trabalhista. Assim, as ferrovias necessitam ser subdividida em trechos, para cada qual são designadas bases e/ou pontos de troca e um conjunto de maquinistas. Cada maquinista realiza apenas uma viagem por escala, entre pontos de troca contínuos, sendo um deles num geral, sua base.

No Brasil o trabalho dos maquinistas é regulamentado pelo Título III, Capítulo I, Seção V da Consolidação das Leis Trabalhistas (CLT) (Brasil, 1943). A CLT define que:

- A escala de trabalho, quando possível, deverá ser de 8 horas, não ultrapassando 12 horas, exceto em caso de acidentes, onde poderá ter-se a carga de trabalho elevada a qualquer número de horas;

- Após cada jornada de trabalho deverá haver um descanso mínimo de 10 horas contínuas, respeitando-se ainda a questão do descanso semanal;

- Quinzenalmente o total de horas de serviço noturno não poderá ser superior às de serviço diurno;

- A escala de "sobreaviso" deverá ser no máximo de vinte e quatro horas;

- A escala de prontidão deverá ser no máximo, de doze horas. 
Portanto, para atender a estas leis e acordos de trabalho específicos, é necessário que os maquinistas conduzam os trens em trechos específicos e pré-determinados, conduzindo somente um trem a cada escala. Sendo assim, diariamente, com base na programação dos trens elaborada previamente, e a disponibilidade dos maquinistas em suas respectivas escalas, aloca-se cada um dos maquinistas a estes trens programados, de maneira a evitar-se ao máximo a geração de custos com horas- extras, horas de prontidão e horas de sobreaviso.

Tabela 1: Concessões ferroviárias no Brasil, e devidas malhas

\begin{tabular}{|c|c|c|c|c|c|}
\hline \multirow{3}{*}{$\begin{array}{l}\text { Malhas } \\
\text { Regionais }\end{array}$} & \multirow[b]{3}{*}{ Ferrovia } & \multirow{2}{*}{\multicolumn{3}{|c|}{ Bitola }} & \multirow[b]{3}{*}{$\begin{array}{l}\text { Total } \\
\text { (km) }\end{array}$} \\
\hline & & & & & \\
\hline & & $\begin{array}{c}\text { Larga } \\
(\mathrm{km})\end{array}$ & $\begin{array}{c}\text { Métrica } \\
(\mathbf{k m})\end{array}$ & \begin{tabular}{|c|} 
Mista \\
$(\mathbf{k m})$
\end{tabular} & \\
\hline MA/PA & Estrada de Ferro Carajás & 978 & & & 978 \\
\hline PR & Estrada de Ferro Paraná Oeste & & 248 & & 248 \\
\hline ES/MG & Estrada de Ferro Vitória Minas & & 873 & 22 & 895 \\
\hline Centro Leste & Ferrovia Centro Atlântica & 3 & 7.089 & 131 & 7.223 \\
\hline Norte Sul & Ferrovia Norte Sul - Tramo Central & 856 & & & 856 \\
\hline Norte Sul & Ferrovia Norte Sul - Tramo Norte & 745 & & & 745 \\
\hline Tereza Cristina & Ferrovia Tereza Cristina & & 163 & & 163 \\
\hline Nordeste & Ferrovia Transnordestina - FTL & & 4.275 & 20 & 4.295 \\
\hline Sudeste & MRS & 1.613 & & 73 & 1.686 \\
\hline MS/MT & Rumo Malha Norte & 735 & & & 735 \\
\hline Oeste & Rumo Malha Oeste & & 1.973 & & 1.973 \\
\hline Paulista & Rumo Malha Paulista & 1.544 & 242 & 269 & 2.055 \\
\hline \multirow[t]{2}{*}{ Sul } & Rumo Malha Sul & & 7.223 & & 7.223 \\
\hline & Total & 6.474 & 22.086 & 515 & 29.075 \\
\hline
\end{tabular}

\section{REVISÃO BIBLIOGRÁFICA}

Roth et al. (2018) trataram em seu artigo sobre o desenvolvimento de um modelo de otimização para apoiar a programação de equipagem da BNSF Railway, uma das maiores companhias ferroviárias dos Estados Unidos, uma vez que o custo com equipagem era o maior entre todos os custos da companhia. À época, o trabalho fora motivado pelo desejo da companhia de substituir seu processo, que era manual, por uma abordagem sistemática e efetiva, uma vez que a metodologia utilizada até então não capturava adequadamente todas as opções e restrições operacionais que surgiam no dia-a-dia, como por exemplo, a incerteza nos horários de trem. Nos estudos foram considerados trechos de destacamento com duas estações, uma considerada como sede da equipagem (casa) e outra como ponto final do percurso. O horizonte de planejamento dos trens que passariam por aquele destacamento utilizado no modelo foi de 48 horas, com especificação de direção de movimento e os horários em que cada trem entraria e sairia do trecho. Além disto, era sabida a localização inicial e o status de repouso de cada membro da equipagem designado ao destacamento. Então, quando um trem adentrasse a um destacamento, fosse qual fosse à direção, a equipagem designada para a outra estação do destacamento desembarcaria naquela estação e descansaria antes de voltar à estação de onde veio. Dependendo da intensidade do tráfego de trens nas duas direções, uma estação poderia ter um número maior de equipagem disponível em relação ao número necessário para operar os trens programados. Nestes casos, a equipagem poderia ser realocada de uma estação para a outra. Isto poderia ser feito em um trem de carga programado, em um trem de passageiros ou em táxi. Para acomodar picos de tráfego, a ferrovia manteria uma lista de equipagem extra na estação sede, a qual só poderia 
ser utilizada quando não houvesse equipagem regular disponível. As decisões de atribuição de equipagem deveriam atender a vários requisitos, garantindo que a cada um dos trens programados fosse atribuído uma equipagem. As conexões em cada estação deveriam cumprir ainda as exigências apropriadas do descanso. Em alguns destacamentos, aplicou-se a regra de "primeiro a entrar, primeiro a sair" (FIFO): para cada ocupação, a equipagem deveria partir da estação na mesma ordem em que chegou. A princípio, para a resolução do problema, utilizou-se uma formulação de programação linear inteira com três principais conjuntos de variáveis de decisão, os quais seguem: variáveis inteiras representando os fluxos de tripulantes nos arcos de viagem, variáveis binárias que indicavam quais arcos de conexão seriam selecionados e variáveis binárias que indicariam quais arcos de viagem de táxi seriam selecionados. A função objetivo minimizaria o custo total com a equipagem durante o horizonte de planejamento, os coeficientes das variáveis de decisão nesta função forneceriam a modelagem de despesas, incluindo pagamentos de tripulação, custos fixos e custos para o uso de equipagens extras, e as restrições para captura adequada dos custos fixos de táxi e atendimento aos requisitos operacionais, como sobreposição de equipes na escala e restrições ao uso de equipagem extra. Porém, o grande número de restrições necessárias para modelar as regras do problema contribuiu para o alcance dos longos tempos computacionais para resolução dos problemas, além de não levar em conta a incerteza dos horários dos trens. Com a incorporação desta incerteza, aumentou-se grandemente a dificuldade do problema e os tempos de solução. Portanto, mudaram o foco para o desenvolvimento de um método heurístico. A efetividade da heurística envolveu versões conclusivas de ambos os algoritmos, exatos e heurísticos, como uma amostra de 1.728 conjuntos de dados reais e comparou os tempos de execução e os custos de solução para cada instância. A heurística encontrou uma solução ótima em todos os casos, exceto $(99,2 \%)$ e aproximadamente $2 \%$ de otimalidade em todos os casos $(99,9 \%)$.

Mazioli e Rosa (2018) tratam de um estudo de caso referente à Estrada de Ferro Vitória a Minas (EFVM), ferrovia de carga que atende aos Estados do Espírito Santo e Minas Gerais, no Brasil. A EFVM conta com seis destacamentos distribuídos por sua malha, cada qual com seu quadro de maquinistas. Tais maquinistas conduzem os trens em trechos específicos, ou seja, partindo de sua sede com destino conhecido e conduzindo apenas um trem por escala. Após o período de descanso, o maquinista volta para a escala para conduzir um novo trem no mesmo trecho da escala anterior. Para testar o modelo matemático proposto, Maziole e Rosa (2018) elaboraram quatro instâncias, onde foram variados os números de maquinistas, destacamentos, trens e escalas. O modelo matemático proposto tomou como base o Problema de Roteamento de Veículos, com Múltiplos Depósitos e com Múltiplas Viagens (PRV-MDMV) (Braekers et al., 2016), onde, cada maquinista é um depósito e existe um depósito virtual correspondente para ele. Em cada depósito, é considerado que existe um único veículo que inicia uma viagem para atender clientes (trens). Uma viagem representa a designação do maquinista e é limitada ao tempo máximo de permanência do maquinista no trem. Um maquinista pode ou não atender trens em uma viagem e, caso o maquinista não atenda nenhum, é considerado que o tempo de viagem do veículo igual ao período de uma escala de trabalho do maquinista, isto no modelo é visto como uma designação nula. Ao fim da viagem o veículo/maquinista retorna para seu respectivo depósito virtual e, no caso do problema de designação de maquinistas, o veículo deve ficar parado até o início de outra viagem, representando o descanso obrigatório dos maquinistas entre escalas. A função objetivo representa a soma das horas extras trabalhadas por todos os maquinistas. Para resolver o modelo matemático proposto, Mazioli e Rosa (2018) utilizaram o solver IBM CPLEX. Os resultados alcançados pelo CPLEX para as quatro instâncias testadas alcançaram a solução ótima. $\mathrm{O}$ modelo matemático proposto mostrou-se uma ferramenta 
aplicável a um problema real, com respostas em um curto espaço de tempo, respeitando legislações nacionais e normas internas da empresa.

Suyabatmaz e Sahin (2015) em seu artigo descreveram um problema que otimizaria o tamanho da tripulação necessária para operação da Turkish Railways baseada em um problema de fluxo de rede de custo mínimo. No artigo eles compararam o desempenho computacional de duas formulações: a formulação baseada em caminho e a formulação baseada em arco, de onde ambos os planejamentos de tripulação viáveis foram extraídos como caminhos de origem-destino de uma rede que representava os dados de um problema. Neste caso os nós representavam espaço e tempo (a estação e a hora de partida), e os arcos representavam os eventos de agendamento (início, termino, repouso e folga) da tripulação. A função objetivo do problema tinha como premissa minimizar o número de membros da tripulação para determinada escala de trabalho. Primeiramente eles verificaram a qualidade das soluções obtidas com a formulação do problema baseadas em caminho (RCCP-C) usando o algoritmo de geração de colunas e linhas (CRG) e a ideia da heurística para obter soluções inteiras. Observou-se que o algoritmo CRG era capaz de resolver a relaxação do problema de Programação Linear LP para otimalidade dentro de um tempo computacional razoável $(3600 \mathrm{~s})$ quando o problema era pequeno. Porém, à medida que o problema ia aumentando em função do horizonte de planejamento e número de folgas, por exemplo, o limite de tempo de 1 hora era atingido antes mesmo que a condição de término fosse satisfeita. Neste caso, em apenas 50\% dos problemas teste foi possível encontrar a solução ótima em até 1 hora. Para a formulação baseada em arco, foi possível resolver não só o relaxamento de LP, mas também a otimização do problema num tempo razoável. Todos os problemas do teste, até a otimização, foram resolvidos em até 2 minutos. Sendo assim, do ponto de vista metodológico, Suyabatmaz e Sahin (2015) concluiram que ambas as abordagens de solução, tanto a formulação baseada em caminho usando algoritmo CRG quanto a formulação baseada em arco, seriam capazes de gerar soluções inteiras. Entretanto a formulação baseada em arco mostrou-se melhor não apenas pela qualidade da solução, mas também com relação ao tempo de solução computacional.

Hanafi e Kozan (2014) desenvolveram um modelo matemático para a programação de tripulação (um maquinista e um auxiliar) de viagens em trens cargueiros, com horários fixos e pré-determinados do início ao fim, da Queensland Rail em Brisbane, Austrália. O objetivo era minimizar o número de tarefas da tripulação, reduzindo os tempos de transição ociosos, incluindo o período de tempo de paradas. $\mathrm{O}$ estudo considerou que haveria locais de destacamento ao longo de todo o trecho e que em cada um destes destacamentos haveria equipes alocadas. Cada segmento de viagem consistiria em uma sequência de viagens que deveriam ser atendidas. Devido ao grande número de variáveis de decisão e restrições levantadas para a resolução do problema, foi utilizado um modelo meta-heurístico em duas fases: a primeira fase construiu soluções iniciais por uma heurística construtiva e a segunda fase melhorou as soluções obtidas pela aplicação de um Simulated Anealling, onde o algoritmo de pesquisa foi aplicado para gerar um cronograma ótimo ou quase ótimo. $\mathrm{O}$ desempenho dos algoritmos propostos foi avaliado pela aplicação de experimentos computacionais em instâncias de teste geradas aleatoriamente. Os resultados mostraram que as abordagens propostas obtiveram soluções quase ótimas em um tempo computacional razoável para um problema de grande porte.

Constantino (1997) desenvolveu em sua tese de doutorado um trabalho cujo objetivo era o de apresentar uma sistemática mensal para o planejamento de escalas de trabalho para maquinistas de trem de carga, levando em conta além da otimização da questão das horas 
trabalhadas, o nível de satisfação de cada condutor. Este foi medido por meio de uma função utilidade ajustada através de técnicas de preferência declarada, onde esta função faz uma ponderação dos principais fatores que causam maior atratividade na escala de trabalho sob a ótica do condutor. O planejamento de escalas foi resolvido em duas etapas: na primeira obteve-se uma escala cíclica otimizada, particionada em programações. Cada programação correspondeu a uma sequência de dias de trabalho entre duas folgas consecutivas. Para resolver este problema, Constantino (1997) duas formas: uma foi uma formulação matemática baseada no modelo set covering e a outra foi um algoritmo heurístico baseado no problema de atribuição. A segunda etapa consistiu em alocar-se as escalas de trabalho aos maquinistas, de forma que o nível de satisfação fosse equitativamente distribuído. A atribuição foi feita levando-se em consideração o nível de satisfação do histórico de trabalho de cada um, e também, o nível de satisfação da escala futura. O problema foi comparado a um problema do caixeiro viajante. A cada iteração foram resolvidos dezenas de problemas de atribuição com gargalo, definidos por uma matriz de utilidades.

Janacek et al. (2017) estudaram o ajuste de cronogramas de recursos, humano e de material rodante, da Netherlands Railways (NS), a maior operadora ferroviária de passageiros dos Países Baixos. Como premissas, adotou-se que as viagens dos trens ocorreriam entre pontos de apoio. Um ponto de apoio era uma estação onde o maquinista poderia mudar de uma composição para a outra. Cada uma destas "mudanças" foi tratada como uma tarefa. Uma viagem poderia conter várias tarefas, e a soma das várias tarefas executadas por um maquinista num dia de trabalho foi chamado de dever. As mudanças entre pontos de apoio também poderiam ser feitas em outros modos de transporte, por isso, também deveriam ser consideradas nos conjuntos das variáveis. O dever deveria começar e terminar na mesma base do maquinista. Para evitar um grande aumento no tempo de resolução computacional, a reprogramação foi permitida apenas para um número limitado de restrições que inviabilizavam os cronogramas de recursos, como ajustes de cronograma, reescalonamento de material rodante e reescalonamento da equipagem. $\mathrm{O}$ algoritmo por eles desenvolvido baseou-se em técnicas de geração de colunas combinadas com as heurísticas de Lagrange, tratadas por GREEDY. A função objetivo minimizou o desvio do cronograma planejado da tripulação, as penalidades por tarefas canceladas, e as penalidades por atrasos.

\section{CONCLUSÕES}

Foi possível observar a grande quantidade de artigos, dissertações e teses, além da literatura tradicional, disponível para consulta e desenvolvimento de trabalhos técnico-científicos na área de planejamento de escalas de motoristas (ou maquinistas, ou pilotos, ou tripulação). A possibilidade de utilização das diversas metodologias e respectivos modelos matemáticos, abre um grande leque no desenvolvimento de ferramentas computacionais para a solução de problemas enfrentados pelas empresas de transporte no Brasil. A informatização/automatização dos processos, como no caso da BNSF Railway estudado por Roth et al. (2018), poderia trazer às Companhias brasileiras economia de milhares, ou até milhões de Reais por ano.

A escolha da metodologia, bem como do(s) modelo(s) matemático(s) a ser(em) utilizado(s), dependerá do tipo de problema a ser abordado (grande, pequeno, muitas variáveis, poucas variáveis) e respectivas características operacionais (legislações, acordos sindicais e normativas das empresas). Num geral os de abordagem heurística apresentaram um melhor desempenho, principalmente na questão do tempo de processamento das respostas. Isso se deveu principalmente ao tamanho dos problemas, quantidade de variáveis, restrições e iterações a serem resolvidas que geralmente são bem elevados. 
Para um futuro trabalho, é possível mesclar-se as diferentes metodologias para, além da minimização dos custos com horas-extras e tempos ociosos de parada, julgar-se se o dimensionamento inicial da equipe está correto. Em todos os casos estudados neste artigo, houve otimização dos processos de planejamento das escalas.

\section{REFERÊNCIAS BIBLIOGRÁFICAS}

[1] ANTF - Associação Nacional dos Transportadores Ferroviários (2019). Disponível em: https:// www.antf.org.br/informacoes-gerais/. Acessado em: 07/05/2019.

[2] Constantino, Ademir Aparecido (1997). "OTIMIZAÇÃO DE ESCALA DE TRABALHO PARA CONDUTORES DE TREM: SEQUENCIAMENTO DE TAREFAS E ALOCAÇÃO BASEADA EM PREFERÊNCIA DECLARADA", [s.d.], 154.

[3] Hanafi, Rosmalina, e Erhan Kozan. "A Hybrid Constructive Heuristic and Simulated Annealing for Railway Crew Scheduling". Computers \& Industrial Engineering 70 (abril de 2014): 11-19. https://doi.org/ 10.1016/j.cie.2014.01.002.

[4] Hoffmann, Kirsten, e Udo Buscher. "Valid Inequalities for the Arc Flow Formulation of the Railway Crew Scheduling Problem with Attendance Rates". Computers \& Industrial Engineering 127 (janeiro de 2019): 1143-52. https://doi.org/10.1016/j.cie.2018.05.031.

[5] Janacek, Jaroslav, Michal Kohani, Matyas Koniorczyk, e Peter Marton. "Optimization of Periodic Crew Schedules with Application of Column Generation Method". Transportation Research Part C: Emerging Technologies 83 (outubro de 2017): 165-78. https://doi.org/10.1016/j.trc.2017.07.008.

[6] Jütte, Silke, e Ulrich W. Thonemann. "Divide-and-Price: A Decomposition Algorithm for Solving Large Railway Crew Scheduling Problems". European Journal of Operational Research 219, no 2 (junho de 2012): 214-23. https://doi.org/10.1016/j.ejor.2011.12.038.

[7] Mazioli, Franco Collodetti, e Rodrigo de Alvarenga Rosa. "PLANEJAMENTO DA DESIGNAÇÃO DE MAQUINISTAS A TRENS DE VIAGEM DE LONGA DISTÂNCIA EM FERROVIAS DE CARGA", [s.d.], 12.

[8] Roth, Brian, Anantaram Balakrishnan, Pooja Dewan, April Kuo, Dasaradh Mallampati, e Juan Morales. "Crew Decision Assist: System for Optimizing Crew Assignments at BNSF Railway". Interfaces 48, $\mathrm{n}^{\circ} 5$ (outubro de 2018): 436-48. https://doi.org/10.1287/inte.2018.0963.

[9] Şahin, Güvenç, e Birol Yüceoğlu. "Tactical Crew Planning in Railways". Transportation Research Part E: Logistics and Transportation Review 47, $\mathrm{n}^{\circ} 6$ (novembro de 2011): 1221-43. https://doi.org/10.1016/j.tre.2011.05.013.

[10] Suyabatmaz, Ali Çetin, e Güvenç Şahin. "Railway Crew Capacity Planning Problem with Connectivity of Schedules". Transportation Research Part E: Logistics and Transportation Review 84 (dezembro de 2015): 88-100. https://doi.org/10.1016/j.tre.2015.10.003.

[11] Veelenturf, Lucas P., Daniel Potthoff, Dennis Huisman, e Leo G. Kroon. "Railway Crew Rescheduling with Retiming". Transportation Research Part C: Emerging Technologies 20, $\mathrm{n}^{\circ} 1$ (fevereiro de 2012): 95-110. https://doi.org/10.1016/j.trc.2010.09.008.

[12] Walker, Cameron G., Jody N. Snowdon, e David M. Ryan. "Simultaneous Disruption Recovery of a Train Timetable and Crew Roster in Real Time". Computers \& Operations Research 32, no 8 (agosto de 2005): 2077-94. https://doi.org/10.1016/j.cor.2004.02.001. 